\title{
Preparation, Characterization, and Drug-Release Properties of Poly( $N$-isopropylacrylamide) Microspheres Having Poly(Itaconic Acid) Graft Chains
}

\author{
Betül Taşdelen, ${ }^{1}$ Nilhan Kayaman-Apohan, ${ }^{2}$ Zülal MisırlI, ${ }^{3}$ Olgun Güven, ${ }^{4}$ Bahattin M. Baysal ${ }^{5,6}$ \\ ${ }^{1}$ Chemistry Department, Çekmece Nuclear Research and Training Center, 34831, P.O. Box 1, Istanbul, Turkey \\ ${ }^{2}$ Department of Chemistry, Marmara University, 81040 Göztepe, Istanbul, Turkey \\ ${ }^{3}$ Advanced Technologies Research and Development Center, Boğaziçi University, 80815 Bebek, Istanbul, Turkey \\ ${ }^{4}$ Department of Chemistry, Hacettepe University, 06532 Beytepe, Ankara, Turkey \\ ${ }^{5}$ Department of Chemical Engineering, Boğaziçi University, 80815 Bebek, Istanbul, Turkey \\ ${ }^{6}$ TUBITAK Marmara Research Center, 41470 Gebze, Kocaeli, Turkey
}

Received 9 March 2004; accepted 1 October 2004

DOI 10.1002/app.21777

Published online in Wiley InterScience (www.interscience.wiley.com).

\begin{abstract}
An inverse suspension polymerization method for the preparation of thermoresponsive hydrogel microspheres based on $N$-isopropylacrylamide was described in this article. The polymerization reaction was carried out at $200 \mathrm{rpm}$ stirring rate and the microspheres obtained were in the size range of $71-500 \mu \mathrm{m}$ in the swollen states. The particles were sieved by using ASTM sieves. The selected fraction $(180-250 \mu \mathrm{m})$ of poly( $N$-isopropylacrylamide) (PNIPAAm) microspheres was used for radiation-induced modification with itaconic acid (IA) to obtain PNIPAAm/poly(itaconic acid) graft copolymer. Viagra and lidocaine were used as model drugs for the investigation of
\end{abstract}

controlled-release behavior of the microspheres. Incorporation of IA graft chains onto microspheres enhanced significantly the uptake of both drugs and further controlled release at specific $\mathrm{pH}$ values. The release studies showed that some of the basic parameters affecting the drug-loading and -release behavior of the microspheres were $\mathrm{pH}$, temperature, particle size, and chemical nature of drug. (c) 2005 Wiley Periodicals, Inc. J Appl Polym Sci 97: 1115-1124, 2005

Key words: microspheres; $N$-isopropylacrylamide; itaconic acid; drug release

\section{INTRODUCTION}

Polymeric hydrogels have found extensive applications, ranging from on-off switching materials, drug delivery systems, immobilization technologies, mass separations, and cell culturing. ${ }^{1-8}$ Available in various physical forms, such as powders, discs, or microspheres, these hydrogels are generally glassy in the dehydrated state, but swell to become elastic gel upon water penetration. In drug-delivery applications, the entrapped drug diffuses through the swollen network into the surrounding aqueous medium. Various parameters including particle size, size distribution, mesh size, swellability, chemical structure, and reactive sites are considered to describe the overall performance of microspheres in drug-release applications.

Correspondence to: B.M. Baysal (bmbaysal@hotmail.com). Contract grant sponsor: TUBITAK.

Contract grant sponsor: Marmara Research Center.

Contract grant sponsor: Turkish- Macedonion Science and Technology Program for 2001-2003.

Contract grant sponsor: Çekmece Nuclear Research and Training Center.

Journal of Applied Polymer Science, Vol. 97, 1115-1124 (2005) (C) 2005 Wiley Periodicals, Inc.
Poly(N-isopropylacrylamide) (PNIPAAm) and its copolymers were studied in the development of temperature-modulated drug delivery systems. ${ }^{9,10}$ PNIPAAm is soluble in cold water, but is not soluble in hot water, and it exhibits a lower critical solution temperature behavior (LCST) at around $32-34^{\circ} \mathrm{C} .{ }^{11}$ Hydrogels composed of PNIPAAm and its copolymers exhibit discrete and reversible volume change in response to infinitesimal changes in temperature. ${ }^{12}$ The LCST of PNIPAAm can be modified by copolymerization with certain comonomers. Hydrophilic comonomers increase the critical temperature so it becomes closer to the human body temperature. ${ }^{13}$

In more recent years, a series of articles was published by Güven and coworkers who synthesized new hydrogels from the copolymers of acrylamide and diprotic itaconic and maleic acid and showed that the use of even very small quantities of diprotic acid proved to impart remarkable properties to the hydrogels of starting monomers and/or homopolymers. ${ }^{14-16}$ The incorporation of acidic moieties into base polymeric structures for the synthesis of microspheres and hydrogels was mostly carried out by using acrylic acid. The use of diprotic acids, however, has been shown to impart additional advantages over 
monoprotic acids such as acrylic and methacrylic acids. ${ }^{17}$ Double ionization of itaconic acid (IA) at different $\mathrm{pH}$ values provides stepwise release behavior of specifically adsorbed drugs or other adsorbates by control of $\mathrm{pH}$ of the medium. Doubly-ionized carboxylic groups bring additional capability of chelate formation under certain cases. It was therefore decided to introduce IA groups as dangling chains onto the microspheres made of PNIPAAm.

Recently, we studied radiation-induced synthesis of P(NIPAAm/IA) copolymeric hydrogels. These hydrogels were used in experiments on swelling and diffusion of the model drugs methylene blue, lidocaine, and sildenafil citrate (Viagra). ${ }^{18}$

In the present study, we report a novel method for the preparation of thermoresponsive PNIPAAm microspheres through a modification of the molecular structure consisting of hydrophilic graft chains. We report for the first time the introduction of hydrophilic poly(itaconic acid) (PIA) graft chains into PNIPAAm microspheres by radiation-induced copolymerization technique. The effect of PIA grafts on high drug loading and the rate of drug release are compared with the behavior of pure NIPAAm microsphere.

\section{EXPERIMENTAL}

\section{Materials}

The monomer $N$-isopropylacrylamide (NIPAAm, Aldrich, Milwaukee, WI), the crosslinker $N, N^{\prime}$-methylenebisacrylamide (MBA, Fluka, Switzerland), the initiator ammonium persulfate (APS, Aldrich), and the accelerator $N, N, N^{\prime}, N^{\prime}$-tetraethylmethylenediamine (TEMED, Aldrich) were used as received. IA was purchased from Fluka Chemical Co. Viagra and lidocaine were provided from Istanbul and Marmara University, Faculty of Pharmacology, respectively, as gifts.

\section{Preparation of microspheres}

PNIPAAm microspheres were prepared by inverse suspension polymerization technique. Polymerization of NIPAAm was conducted in a 500-mL round-bottom, four-neck flask, fitted with a mechanical stirrer, a nitrogen inlet, and a pipette outlet. PNIPAAm microspheres were prepared without using an external emulsifier. Paraffin oil was used as the continuous phase, as reported by Park and Hoffman. ${ }^{19}$ The synthesis has been described in detail elsewhere. ${ }^{4}$ Briefly, $200 \mathrm{ml}$ paraffin oil was first introduced into the reactor and stirred at $200 \mathrm{rpm}$ under nitrogen atmosphere; $4.0 \mathrm{~g}$ of the monomer mixture (NIPAAm and MBA) and the initiator APS (40 mg) were dissolved in distilled water $(20 \mathrm{ml})$, and then $\mathrm{N}_{2}$ was bubbled through the solution for $10 \mathrm{~min}$. This solution was transferred into the reactor, and after $1 \mathrm{~min}$, TEMED $(=0.12 \mathrm{ml})$ was added to the mixture to initiate the polymerization. The reaction was allowed to proceed for $3 \mathrm{~h}$ at $22^{\circ} \mathrm{C}$. After polymerization, the microspheres were separated from the oil phase and washed several times with acetone and water. The diameter of the microspheres in the swollen state ranged from 71 to $500 \mu \mathrm{m}$. The particles were sieved by using ASTM sieves, separated, left to dry at room temperature and then further dried at $40^{\circ} \mathrm{C}$ in a vacuum oven overnight. Selected fractions were used for further experiments.

\section{Preparation of PNIPAAm microspheres having PIA graft chains}

The selected fraction of PNIPAAm microspheres between 180 and $250 \mu \mathrm{m}$ size ranges was used for preparing PNIPAAm/PIA graft copolymer by radiationinduced modification technique. One gram of dry PNIPAAm microspheres was put into a $10 \% \mathrm{w} / \mathrm{w}$ aqueous solution of IA and rested at $4^{\circ} \mathrm{C}$ for $24 \mathrm{~h}$ to reach equilibrium swelling., ${ }^{4,5}$ Then, the swollen PNIPAAm microspheres were transferred to small glass tubes for irradiating with a ${ }^{60} \mathrm{Co}$-gamma source at $25^{\circ} \mathrm{C}$ under air at different irradiation doses $(5,24$, and $48 \mathrm{kGy}$ ). After irradiation, the PNIPAAm microspheres having PIA graft chains were immersed into a large excess of water to wash out any unreacted IA. Finally, they were dried in vacuum to constant weight. To obtain IA incorporation quantitatively into the PNIPAAm network, the IA content of the PNIPAAm/ PIA copolymeric microspheres was determined by titration of extract against $\mathrm{NaOH}(0.1 N)$ to phenolphthalein end point. ${ }^{20}$

\section{Characterization}

Polymer characterization

The IR spectra of the microspheres were recorded on a Jasko 5300 Model FTIR spectrophotometer. Samples were ground with a mortar and pestle and mixed with $\mathrm{KBr}$ at a ratio of $1: 100$. The mixture was then pressed into a pellet and spectra were analyzed from 400 to $4000 \mathrm{~cm}^{-1}$.

IR $\left(\mathrm{KBr}, \mathrm{cm}^{-1}\right)(\mathrm{A}) 3435 \mathrm{NH}$ str.,
1640C $=$ Ostr.(amide I),
1564NHbend.(amide II)

(B) $1720 \mathrm{C}=$ Ostr.,

$1629 \mathrm{C}=$ Ostr. (amide I),1550NHbend. (amide II)

Optical micrograph of the PNIPAAm microspheres was obtained by using a Reichert Universal MeF2-type microscope. 


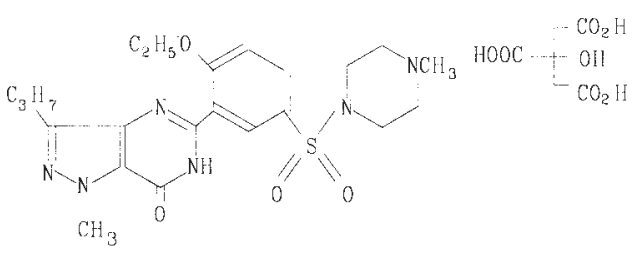

Sildenafil Citrate (Viagra)

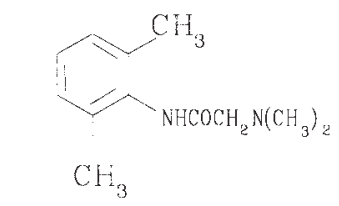

I.idoceine

Figure 1 The chemical formula of the drugs.

Scanning electron micrographs were taken on a Philips XL30 ESEM FEG environmental scanning electron microscope, making it possible to examine materials in their natural, uncoated state at low vacuum around $0.2-1.5 \mathrm{~Pa}$ and at $10 \mathrm{kV}$. During in situ testing of uncoated powders, it is an obvious advantage that charging will not occur. The powder morphologies were imaged and generated in their natural surface characteristics by using a special technique (gaseous low vacuum mode) allowing charge-free, gaseous secondary spraying with air. The release profiles of drugloaded microspheres were determined by using a Shimadzu Model UV-160A spectrophotometer.

\section{Drug-loading and -releasing experiments}

In this work, Viagra (sildenafil citrate) (VG) (MW $=667 \mathrm{~g} / \mathrm{mol})$ and lidocaine (LD) $(\mathrm{MW}=234 \mathrm{~g} / \mathrm{mol})$ were used as model drugs in release experiments (Fig. 1). The dry microspheres were equilibrated in 500 ppm $(\mathrm{mg} / \mathrm{L})$ of $V G$ and $5000 \mathrm{ppm}(\mathrm{mg} / \mathrm{L})$ of $\mathrm{LD}$ solutions, prepared in phosphate buffer, at $\mathrm{pH} 7.4$ at $4^{\circ} \mathrm{C}$ for 1 week, respectively. After incubation, the microspheres were removed from the solution and rinsed in cold buffer. The VG and LD release experiments were carried out by transferring previously incubated drug-loaded microspheres into a vessel containing $50 \mathrm{~mL}$ phosphate buffer at $\mathrm{pH} 7.4$ at $37^{\circ} \mathrm{C}$ at a constant shaking rate. At various times, $3-\mathrm{mL}$ aliquots were drawn from medium to follow VG and LD release and placed again into the same vessel so that the liquid volume was kept constant. VG and LD release were determined spectrophotometrically at 291 and $262 \mathrm{~nm}$, respectively. The controlled release of nonspecifically adsorbed drug was followed at $\mathrm{pH}$ 7.4. The amount of percentage release of drug at $\mathrm{pH} 7.4$ was calculated from the equation:
Release $\%$ of nonspecific adsorbed drug $=$

$$
\left(w_{t} / w_{\text {total }}\right) \times 100
$$

where $w_{t}$ is the weight of released drug at time $t$ and $w_{\text {total }}$ is the total weight of specific and nonspecific adsorbed drug in the gel structure. Buffers at $\mathrm{pH} 5.5$, $\mathrm{pH} 4.0$, and $\mathrm{pH} 2.0$ were used for the controlled release of specifically bonded drug from the gels. The interactions between cationic groups of drugs and carboxyl groups of IA led to specific drug adsorption. The percentage release of specific adsorbed drug was calculated from the equation:

Release $\%$ of specific adsorbed drug

$$
=\left(w_{t} / w_{\mathrm{sp}}\right) \times 100
$$

where $w_{t}$ is the weight of released drug at time $t$ and $w_{\mathrm{sp}}$ is the total weight of specific adsorbed drug in the gel system. After release at $\mathrm{pH} 2$, the gels were immersed in $0.1 \mathrm{~mol} / \mathrm{L} \mathrm{HCl}$ for 2 days to remove any remaining drug in the gel structure. The amounts pertaining to the uptake and release of both drugs at various conditions are collected in Table II.

\section{RESULTS AND DISCUSSION}

PNIPAAm microspheres prepared by inverse suspension polymerization were used for drug adsorption and release experiments. Rigid, transparent, and spherical beads were obtained without using an external emulsifier and, therefore, they are free from impurities affecting the drug delivery process.

Figure 2 shows a typical size distribution of PNIPAAm microspheres in the swollen state. The bar graph indicates that about $64 \%$ of the microspheres were in the range of $180-250 \mu \mathrm{m}$. A representative

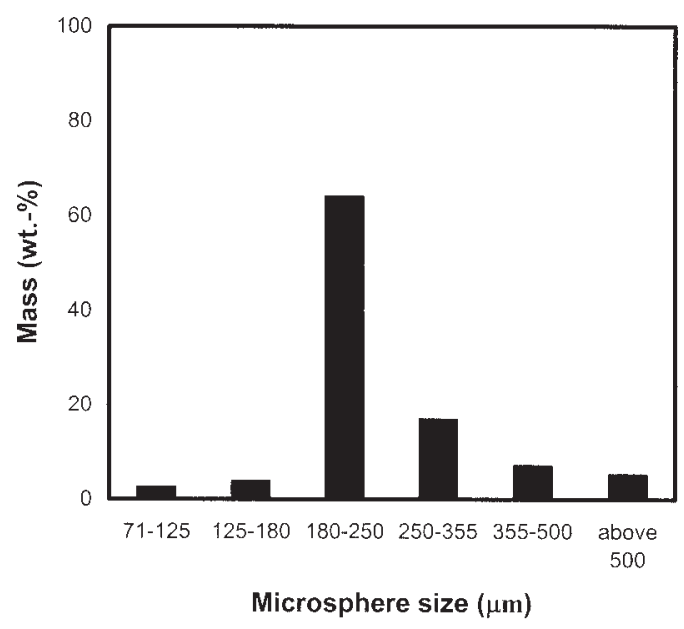

Figure 2 Size distribution of PNIPAAm microspheres in the swollen state. 


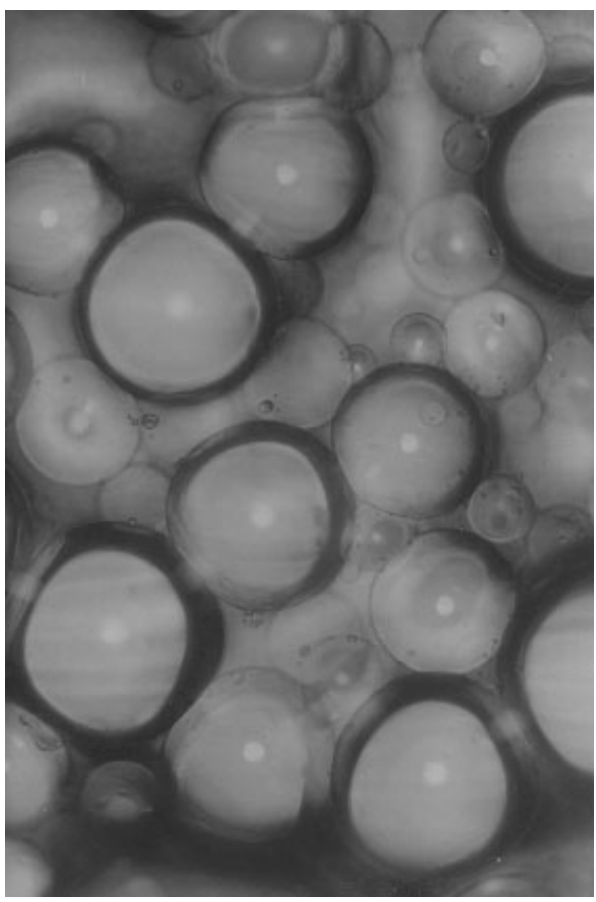

Figure 3 A representative optical micrograph of PNIPAAm microspheres in the swollen state. Magnification, $\times 80$.

optical micrograph of the microspheres is shown in Figure 3.

The PNIPAAm/PIA graft copolymeric microspheres were obtained successfully by using the radiation-induced synthesis. The mol \% of IA in the copolymeric microspheres are calculated and summarized in Table I. As the irradiation dose increases, IA content in the copolymeric gels increases.

FTIR spectroscopy was also used to investigate the grafting of PIA into the PNIPAAm network. Figure 4 shows FTIR spectra of pure PNIPAAm microspheres and PNIPAAm/PIA graft copolymeric microspheres (after extraction) [(PNIPAAm/PIA)-1]. As can be seen in Figure 4(a), pure PNIPAAm microspheres showed a characteristic amide I band at $1640 \mathrm{~cm}^{-1}(\mathrm{C}=\mathrm{O}$ stretching) and amide II band at $1564 \mathrm{~cm}^{-1}(\mathrm{~N}-\mathrm{H}$ bending). Also, only one $\mathrm{N}-\mathrm{H}$ stretching band that is characteristic for secondary amides at $3435 \mathrm{~cm}^{-1}$ can be seen in the FTIR spectrum of PNIPAAm microspheres. In the FTIR spectrum of the graft copolymer with $9.8 \%$ IA content [(PNIPAAm /PIA)-3] [Fig. 4(b)], carbonyl $(\mathrm{C}=\mathrm{O})$ stretching band of acid at $1720 \mathrm{~cm}^{-1}$ appeared. This indicates that the modification of PNIPAAm microspheres was performed successfully.

The environmental scanning electron microscopy (ESEM) images were analyzed comparatively to quantify the particle size and morphology as a function of diameter; images were digitized directly by the image program. The differences between figures were exam- ined for the investigation of drug adsorption behaviors affecting the outlook of PNIPAAm microspheres (180-250 $\mu \mathrm{m})$.

Figure 5(a) shows the ESEM of pure PNIPAAm microspheres in dry form. It is evident that dried PNIPAAm microspheres exhibited spherical shape and smooth topography. These microspheres had a mean particle diameter of $45 \mu \mathrm{m}$ in dry form. Figure 5 (b)s shows the ESEM of the PNIPAAm microspheres after the LD loading. The morphology of LD incorporated spheres was not much different from the blank PNIPAAm microspheres. Figure 5(c) illustrates the more detailed surface morphology of the PNIPAAm microspheres after the LD loading. As shown in Figure $5(a-c)$, there is no noticeable difference among them.

However, these studies concluded that the morphological examination was insufficient for the accurate loading analysis. We will discuss in more detail some aspects of pore ratios in respect to different imaging conditions and specimen preparation.

\section{Controlled release behaviors of microspheres}

Figure $6(a, b)$ shows the effect of particle size on total drug uptake into pure PNIPAAm microspheres in buffer at $\mathrm{pH} 7.4$ and $4^{\circ} \mathrm{C}$. Experiments were performed by using two different kinds of drugs. VG is a weak acid and slightly soluble in water and buffer at $\mathrm{pH}$ 7.4. LD is a water-soluble and slightly basic structure. It was observed that the amount of total drug taken increased with the decrease in microsphere size. The reason for this variation in drug uptake was attributed to the increase of the surface area.

In Table II total drug uptake and equilibrium release amounts for microspheres can be seen. One can see from Table II that the drug-loading capacity of PNIPAAm/PIA gel is higher than PNIPAAm gel. This indicates that incorporation of IA graft chains onto microspheres significantly enhanced the uptake of LD. In the case of pure PNIPAAm microspheres, the drug loading increases as the molecular size of the drug decreases. If the molecular weight of the drug is sufficiently high, it does not penetrate inside the pores.

TABLE I

The Effect of Irradiation Dose on the Mol \% of IA in the Copolymeric Microspheres

\begin{tabular}{lcc}
\hline Gel name & $\begin{array}{c}\text { Irradiation } \\
\text { dose } \\
(\mathrm{kGy})\end{array}$ & $\begin{array}{c}\text { Mole \% IA } \\
\text { in the gel }\end{array}$ \\
\hline PNIPAAm/PIA-1 & 5 & 6.0 \\
PNIPAAm/PIA-2 & 24 & 8.7 \\
PNIPAAm/PIA-3 & 48 & 9.8 \\
\hline
\end{tabular}




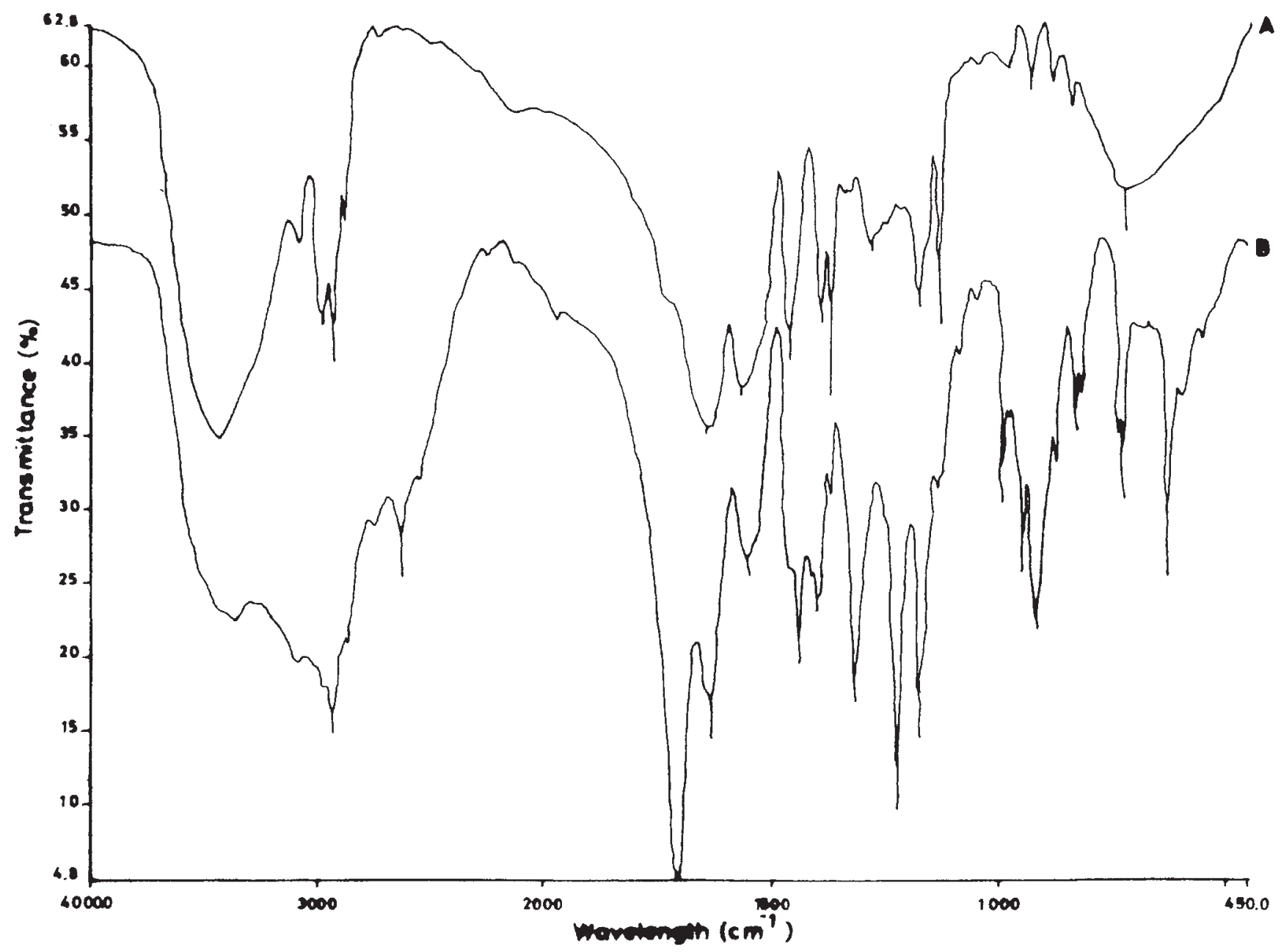

Figure 4 FTIR spectrum of the pure PNIPAAm microspheres (180-250 $\mu \mathrm{m})$ (a), and surface-modified PNIPAAm/PIA graft copolymer, after extraction (b).

Thus, it can be concluded that molecular weight of the drug is one of the determining parameters that affects the drug uptake in addition to specific interactions, drug concentration, degree of gel swelling, and gel mesh size.

Drug-loading efficiency increases for the drugs because of some specific interactions of carboxyl groups of IA with the drugs. Different binding features are responsible for the drug-loading contents. LD has specific interaction with PNIPAAm/PIA gels through electrostatic interactions. How does the adsorption of VG take place? When PNIPAAm/ PIA microspheres were exposed to a VG solution, citrate ions are replaced by PIA ionic groups and thus sildenafil interacts with the microspheres through electrostatic interactions. Also, the adsorption of VG is assumed to take place mostly by ion-dipole-type interactions.

The interactions between cationic groups of the drug and carboxyl groups of IA lead to specific drug adsorption. On the other hand, nonspecific drug adsorption is due to physical inclusion, pore filling action of drugs into completely ionized gel at $\mathrm{pH}$ 7.4, or any $\mathrm{pH}$ above 5.44 that corresponds to a second $\mathrm{pKa}$ of IA. Pore intermolecular forces other than the electrostatic attraction leads to nonspecific drug adsorption. To determine the amount of nonspecific adsorbed drug, microspheres are placed in pH 7.4 phosphate-buffered solution. The release profiles of each drug from PNIPAAm/PIA microspheres in phosphate-buffered solution of $\mathrm{pH} 7.4$ at $37^{\circ} \mathrm{C}$ were shown in Figure $7(\mathrm{a}, \mathrm{b})$, respectively. The release percentage for nonspecific adsorbed drug was higher for pure PNIPAAm microspheres than those for PNIPAAm/PIA microspheres. Although $98 \%$ of VG and $93 \%$ of LD were released from PNIPAAm microspheres, this value decreased to $38 \%$ of VG and $54 \%$ of LD for PNIPAAm/PIA-3. This indicates that specific binding of both drugs is totally due to the presence of IA graft chains. Significant burst effects were not found and release occurred during short periods. 


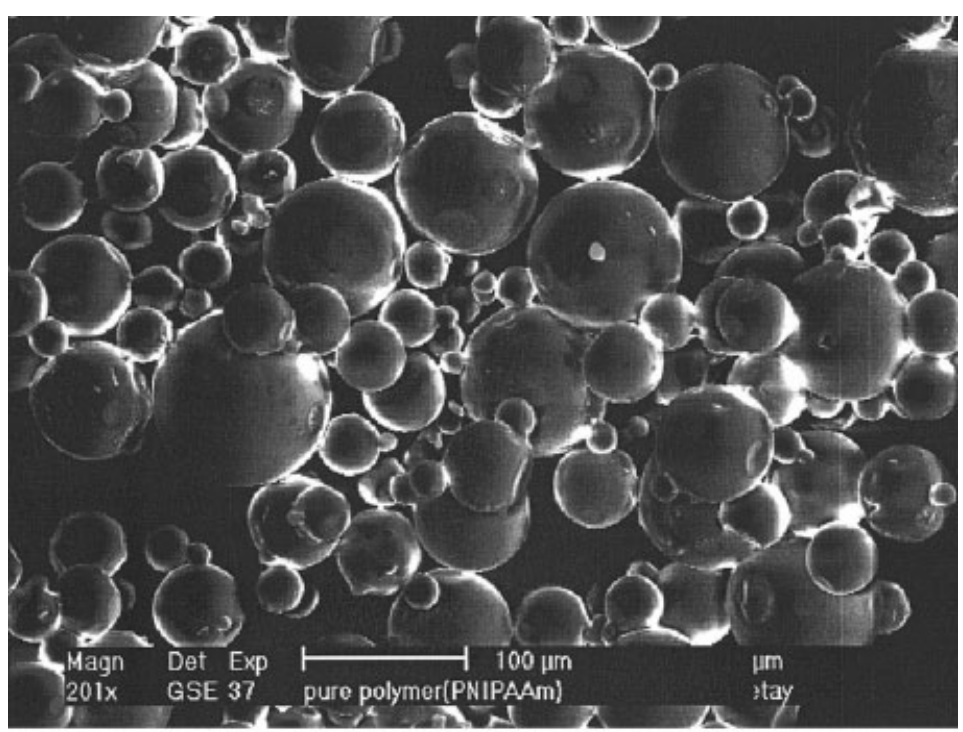

(a)

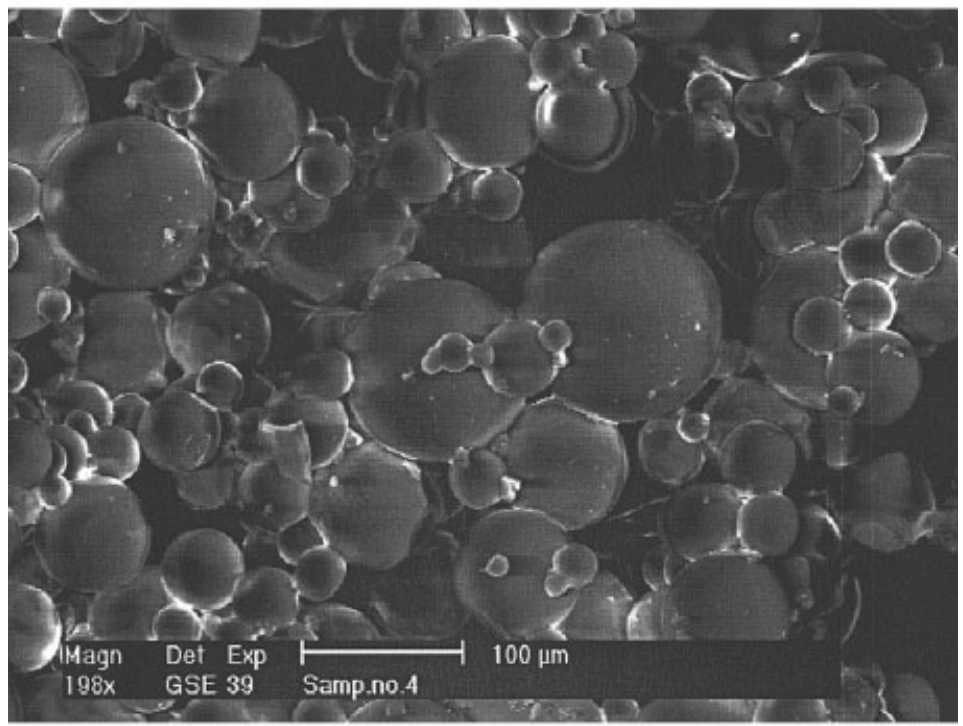

(b)

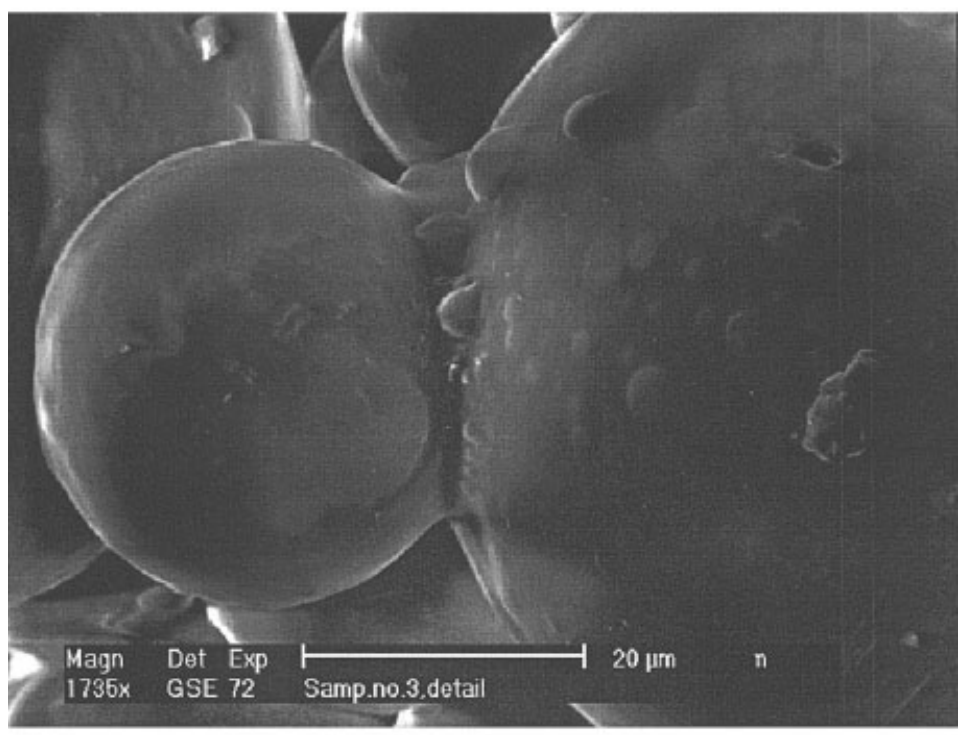

(c)

Figure 5 (a) Environmentally scanning electron micrograph (ESEM) of pure PNIPAAm microspheres. (b) ESEM of the PNIPAAm microspheres after lidocaine loading. (c) The detailed surface morphology of the PNIPAAm microspheres after the LD loading. 


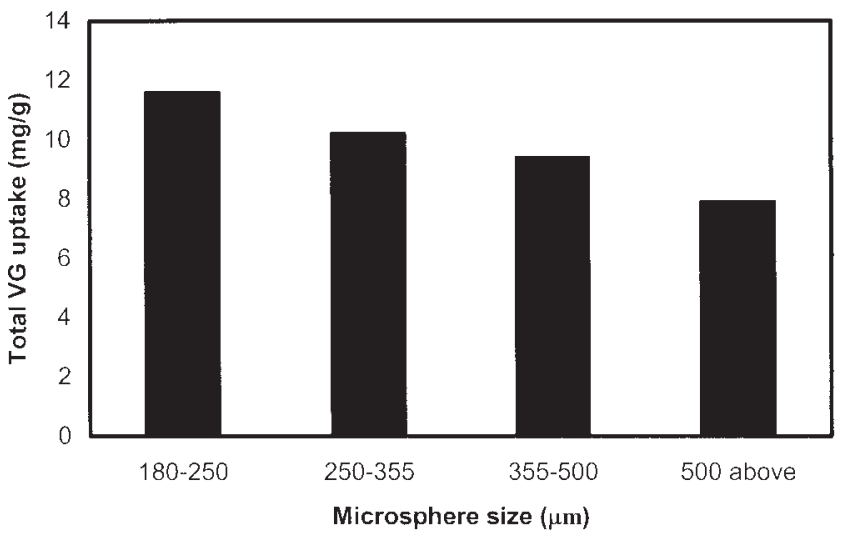

(a)

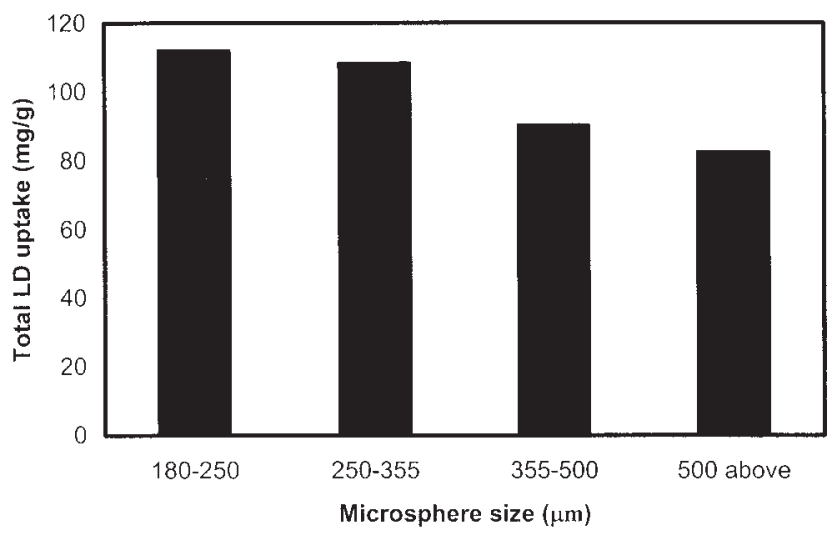

(b)

Figure 6 (a) The effect of particle size on the equilibrium VG release from PNIPAAm microspheres in buffers at pH 7.4 and $37^{\circ} \mathrm{C}$. (b) The effect of particle size on the equilibrium LD release from PNIPAAm microspheres in buffers at pH 7.4 and $37^{\circ} \mathrm{C}$.

The incomplete release of the drugs from PNIPAAm/ PIA microspheres at $\mathrm{pH} 7.4$ was due to specific binding of these drugs to dissociated groups of IA units of the polymer. The difference between the total and nonspecific drug uptake is therefore taken to be equal to the amount of specific adsorbed drug in the microsphere. The controlled release of specific adsorbed drug from PNIPAAm/PIA microspheres was investigated primarily at $\mathrm{pH} 5.5$ because the second dissociation constant $\left(\mathrm{pK}_{\mathrm{a} 2}\right)$ of IA is around $5.44 .^{21}$ The drug release was followed until equilibrium and then the microspheres were transferred into drug-free buffer at $\mathrm{pH} 4\left(\mathrm{pK}_{\mathrm{a} 1}=3.85\right)$. In the release of specific adsorbed drug from the microspheres, one of the anionic carboxylate groups was protonated at $\mathrm{pH}$ 5.5. Then, the first protonation was completed by keeping the spheres at $\mathrm{pH} 4$. Finally, the microspheres were placed into $\mathrm{pH} 2$ buffer for the complete protonation of acid groups since the second $\mathrm{pKa}$ is 3.85 for IA. The percentage specific release of the drug with time is given in Figure 8. The total release percentage was found as 97\% VG and 82\% LD for PNIPAAm/PIA-3 after completion of the release experiments in all buffer solutions. These results illustrate that VG and LD are not completely released and some portions are entrapped within the gel. Because the drugs were initially loaded at a temperature well below the LCST of base material PNIPAAm, in fully swollen state, and release studies were performed at $37^{\circ} \mathrm{C}$ where the PNIPAAm chains were in collapsed state, some portion of the drugs might well have been entrapped in the shrunken part of the spheres.

One of the most attractive features of PNIPAAmbased hydrogels as drug carriers is their intelligent property to external temperature changes. It is important and practical to examine the drug-release data from those PNIPAAm/PIA microspheres at a temperature >LCST, such as body temperature $\left(37^{\circ} \mathrm{C}\right)$. Figure 9 shows the drug-release behavior of each drug from the PNIPAAm/PIA microspheres in buffer at $\mathrm{pH} 7.4$ at two different temperatures (20 and $37^{\circ} \mathrm{C}$ ). Release percentages of each nonspecific adsorbed drug at $37^{\circ} \mathrm{C}$ were lower than those at $20^{\circ} \mathrm{C}$ because of the collapsed nature of PNIPAAm structure at a temperature greater than its LCST $\left(34^{\circ} \mathrm{C}\right) .{ }^{22}$

\section{CONCLUSION}

In this study, PNIPAAm microspheres were prepared by inverse suspension polymerization

TABLE II

The Total Drug Uptake and Equilibrium Release Results

\begin{tabular}{|c|c|c|c|c|c|c|c|}
\hline \multirow[b]{2}{*}{ Gel name } & \multirow{2}{*}{$\begin{array}{l}\text { Drug } \\
\text { name }\end{array}$} & \multirow{2}{*}{$\begin{array}{c}\text { Total uptake } \\
\text { (mg/g dry gel) } \\
\text { pH } 7.4\end{array}$} & \multirow{2}{*}{$\begin{array}{c}m_{\text {nonspecific released }} \\
\text { (mg/g dry gel) } \\
\text { pH } 7.4\end{array}$} & \multicolumn{3}{|c|}{$m_{\text {specific released }}(\mathrm{mg} / \mathrm{g}$ dry gel $)$} & \multirow{2}{*}{$\begin{array}{c}m_{\text {nonreleased }} \\
\text { (mg/g dry gel) }\end{array}$} \\
\hline & & & & pH 5.5 & $\mathrm{pH} 4.0$ & pH 2.0 & \\
\hline PNIPAAm & VG & 11.6 & 11.4 & & - & & 0.2 \\
\hline PNIPAAm/PIA-3 & VG & 38.2 & 7.2 & 11.7 & 12.7 & 5.6 & 1.0 \\
\hline PNIPAAm & LD & 122.2 & 114 & & - & & 8 \\
\hline PNIPAAm/PIA-3 & LD & 201.8 & 119.7 & 42.8 & 2.8 & 1.8 & 34.7 \\
\hline
\end{tabular}




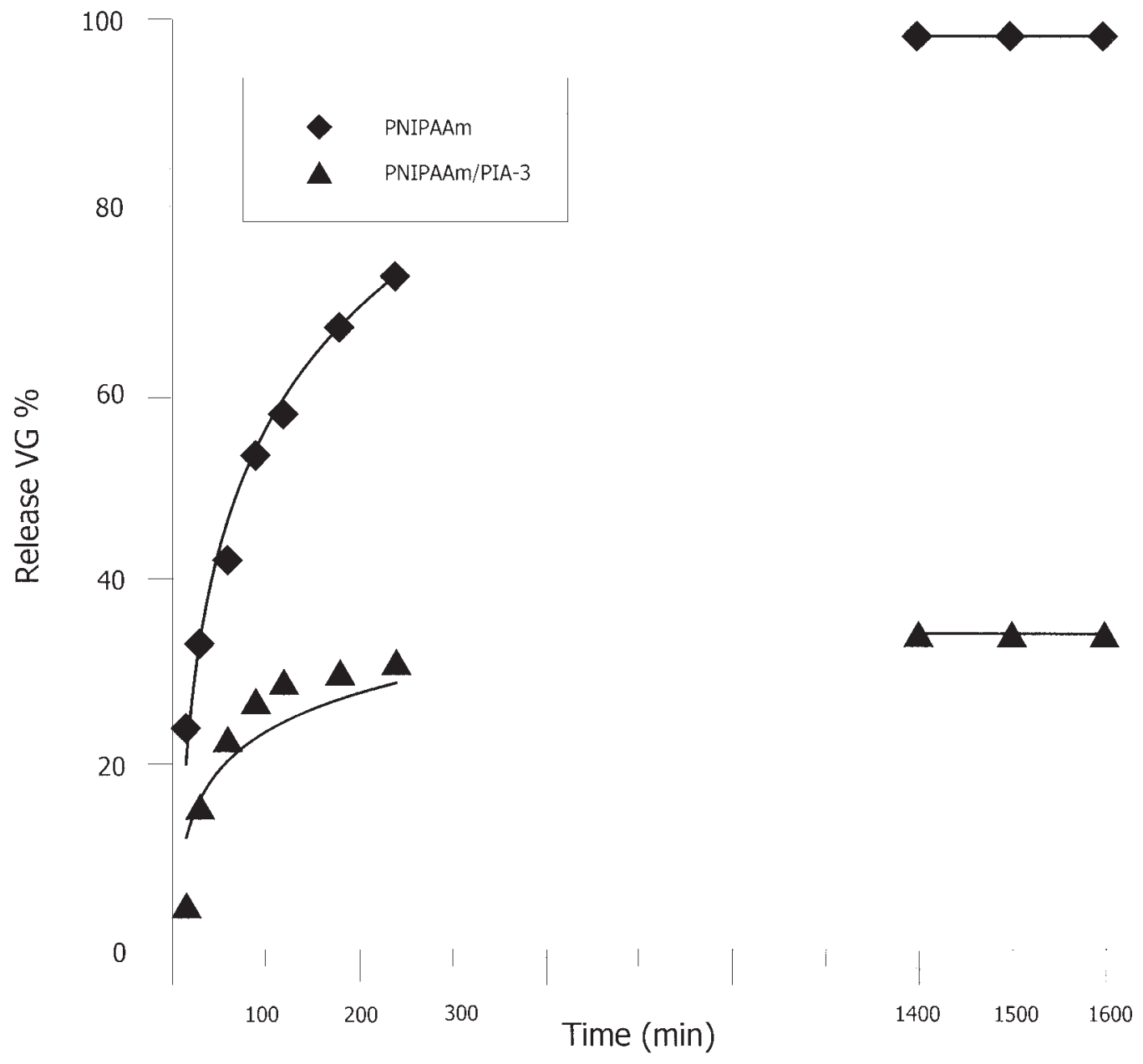

(a)

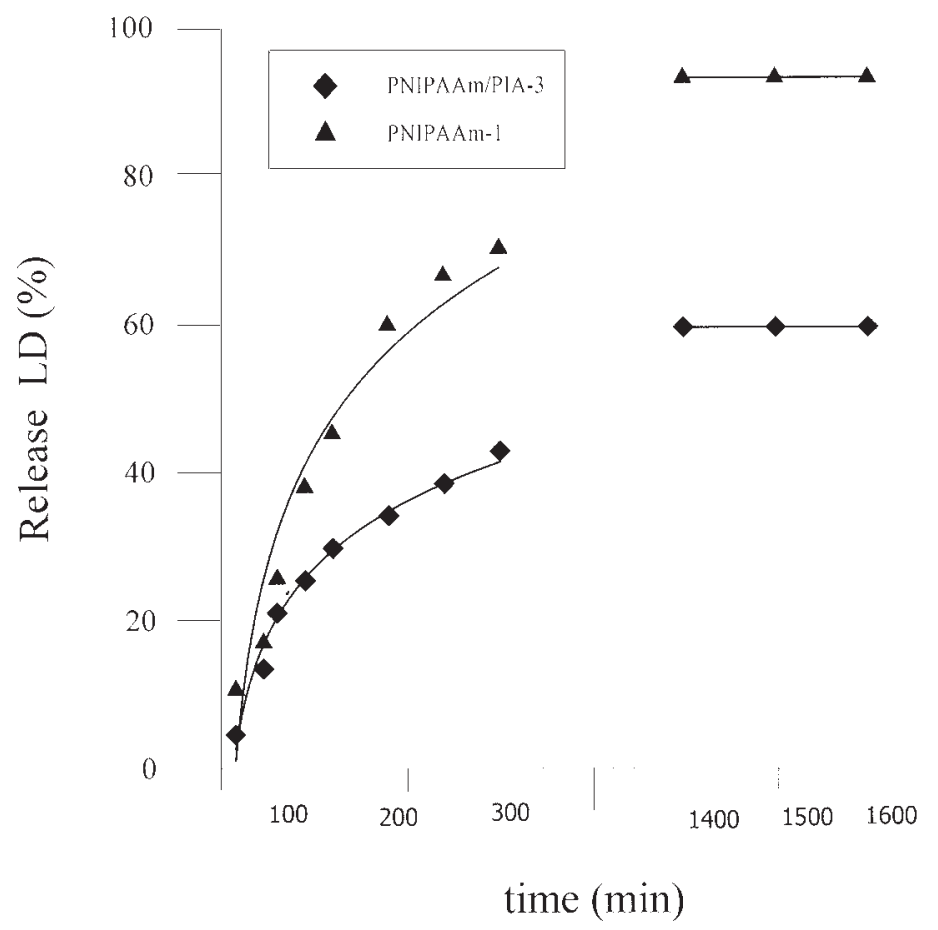

(b)

Figure 7 (a) Release percentage of nonspecific adsorbed VG from PNIPAAm/PIA-3 microspheres in phosphate-buffered solution of $\mathrm{pH} 7.4$ at $37^{\circ} \mathrm{C}$. (b) Release percentage of nonspecific adsorbed LD from PNIPAAm/PIA-3 microspheres in phosphate-buffered solution of $\mathrm{pH} 7.4$ at $37^{\circ} \mathrm{C}$. 


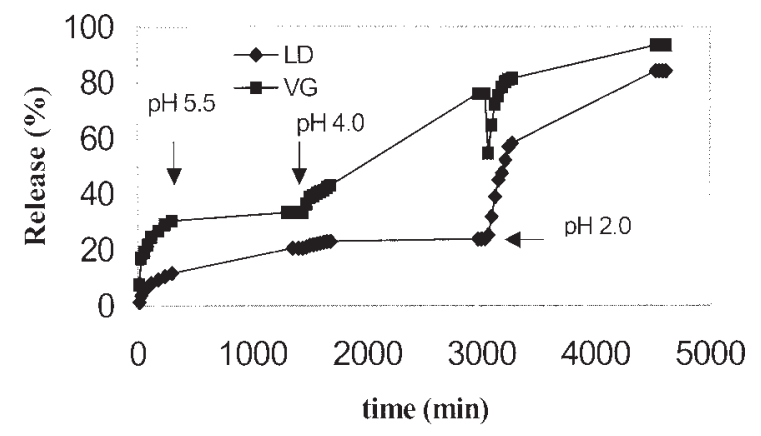

Figure 8 Release percentage of specific adsorbed drug from PNIPAAm/PIA-3 microspheres at $37^{\circ} \mathrm{C}$.

method. Radiation-induced polymerization technique was performed to prepare PNIPAAm/PIA graft copolymeric microspheres. Drug-release behaviors of the microspheres were investigated by using VG and LD as model drugs. It was found that the specific and nonspecific adsorption capacity of microspheres both increase with incorporating IA into the PNIPAAm gel structure. This can be explained by the additional interactions caused by ionic sites and consequent higher swelling capacity of the gels. The release studies show that some of the basic parameters affecting the drug-release behavior of PNIPAAm/PIA microspheres is $\mathrm{pH}$ and temperature of the solution. The other important factors are the water solubility and the molecular size of drugs, and specific interactions of the drug with polymers. To conclude, the microspheres prepared in this study can be considered as potential carriers for the drug-delivery systems and may be used especially as local therapeutic application of cationic drugs under controlled $\mathrm{pH}$ and temperature conditions.

This work was supported by TUBITAK, Marmara Research Center, Turkish-Macedonian Science and Technology Program for 2001-2003 and Cekmece Nuclear Research and Training Center. B.M.B. and O.G. acknowledge support from TUBA-Turkish Academy of Sciences.

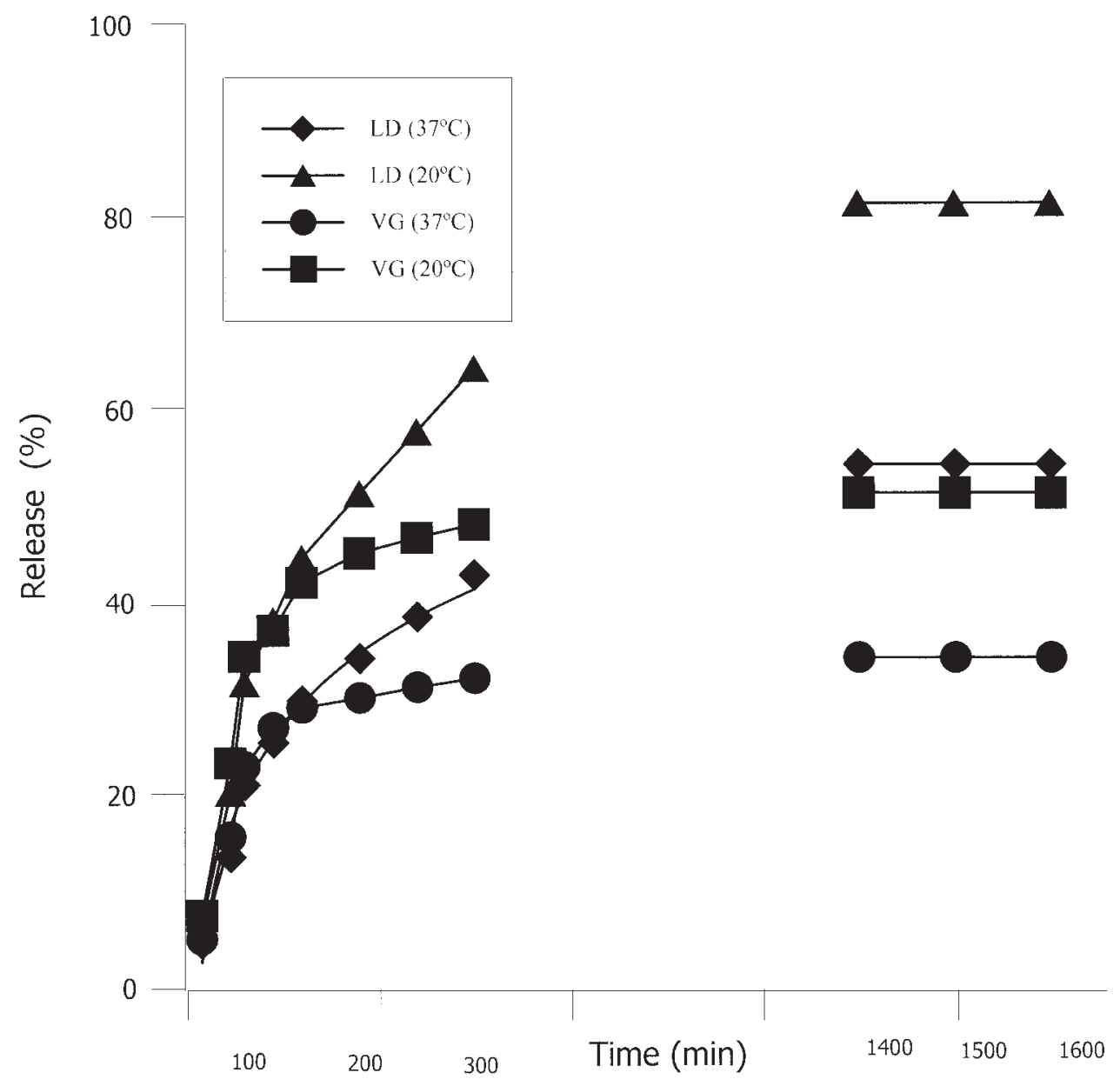

Figure 9 Release percentage of nonspecific adsorbed drug from PNIPAAm/PIA-3 microspheres in phosphate-buffered solution of $\mathrm{pH} 7.4$ at two different temperatures $\left(20\right.$ and $\left.37^{\circ} \mathrm{C}\right)$. 


\section{References}

1. Hoffman, A. S.; Afrassiabi, A.; Dong, L. C. J Controlled Release 1986, 4, 213.

2. Kaneko, Y.; Nakamura, S.; Sakai, K.; Aoyagi, T.; Kikuchi, A.; Sakurai, Y.; Okano, T. Macromolecules 1998, 31, 6099.

3. Kim, C. J.; Lee, P. I. J Appl Polym Sci 1992, 46, 2147.

4. Kayaman, N.; Kazan, D.; Erarslan, A.; Okay, O.; Baysal, B. M. J Appl Polym Sci 1998, 67, 805.

5. D'Emanuele, A.; Dinarvand, R. Polymer 1995, 118, 237.

6. Lee, W.-F.; Yuan, W.-Y. J Appl Polym Sci 2000, 77, 1760.

7. Dinarvand, R.; D'Emanuele, A. J Controlled Release 1995, 36, 221.

8. Lin, S. Y.; Chen, K. S.; Run-Chu, L. Polymer 1999, 40, 6307.

9. Bae, Y. H.; Okano, T.; Kim, S. W. Makromol Chem Rapid Commun 1987, 8, 481.

10. Hoffman, A. S. J Controlled Release 1987, 6, 297.

11. Dušek, K. Responsive Gels: Volume Transitions II; Advances in Polymer Science 110, Springer-Verlag: Berlin, 1993.
12. Hirokawa, Y.; Tanaka, T. J Chem Phys 1984, 81, 6379.

13. Kuckling, D.; Adler, H. P.; Arbdt, K. F.; Ling L.; Habicher, W. D. Macromol Chem Phys 2000, 220, 273.

14. Karadağ, E.; Saraydin, D.; Öztop, H. N.; Güven, O. Polym Adv Technol 1994, 5, 664.

15. Karada ̆̆, E.; Saraydin, D.; Getinkaya, S.; Güven, O. Biomaterials $1996,17,67$.

16. Saraydin, D.; Karadağ, E.; Güven, O. Polym Adv Technol 1994, 6,719 .

17. Güven, O.; Şen, M.; Karadağ, E.; Saraydin, D. Radiation Phys Chem 1999, 56 (4), 381.

18. Taşdelen, B.; Kayaman-Apohan, N.; Güven, O.; Baysal, B. M. J Appl Polym Sci 2004, 91, 911.

19. Park, T. G.; Hoffman, A. S. Biotechnol Prog 1994, 10, 82.

20. Şen, M.; Yakar, A.; Güven, O. Polymer 1999, 40, 2969.

21. Weast, R. C. Handbook of Chemistry and Physics, 53rd ed.; The Chemical Rubber Co., OH, 1972.

22. X. Zheng, D. Wu, C. Chu, Biomaterials to appear. 